\title{
Prospective randomized comparison of a 22G core needle using standard versus capillary suction for EUS-guided sampling of solid pancreatic masses
}

(ㄷ)(1) $(9)$

\author{
Authors \\ Brian R. Weston ${ }^{1}$, William A. Ross ${ }^{1}$, Manoop S. Bhutani ${ }^{1}$, Jeffrey H. Lee ${ }^{1}$, Mala Pande ${ }^{1}$, Andrew B. Sholl ${ }^{2}$, Savitri \\ Krishnamurthy ${ }^{1}$
}

Institutions

1 The University of Texas MD Anderson Cancer Center, Houston, Texas, United States

2 Tulane University School of Medicine, New Orleans, Louisiana, United States

submitted 30.10 .2016

accepted after revision $\quad 6.2 .2017$

\author{
Bibliography \\ DOI https://doi.org/10.1055/s-0043-105492 | \\ Endoscopy International Open 2017; 05: E505-E512 \\ (c) Georg Thieme Verlag KG Stuttgart · New York \\ ISSN 2364-3722
}

Corresponding author

Brian R. Weston, MD, Associate Professor, Department of Gastroenterology, Hepatology and Nutrition, The University of Texas MD Anderson Cancer Center, 1515 Holcombe Blvd. Unit 1466, Houston, TX 77030

Fax: +1-713-563-4408

bweston@mdanderson.org

\section{ABSTRACT}

Background and study aims The optimal technique for sampling pancreatic lesions with a $22 \mathrm{G}$ Procore needle (pc) is unknown. The aims of this study were to evaluate the 22 Gpc using standard suction technique (SST) and ca- pillary suction technique (CST) and compare diagnostic adequacy of $22 \mathrm{Gpc}$ with the standard $25 \mathrm{G}$ needle.

Patients and methods Sixty consecutive patients referred for EUS-FNA of a solid pancreatic mass were prospectively evaluated. All patients underwent 2 passes with a standard $25 \mathrm{G}$ needle for cytologic analysis. The first group of $30 \mathrm{pa}-$ tients underwent a single pass with the $22 \mathrm{Gpc}$ needle using SST for cytology and histology. The second group underwent a single pass with the $22 \mathrm{Gpc}$ needle using CST. The sequence of passes was randomized. The diagnostic adequacy of each pass was graded by 2 cytopathologists blinded to technique and needle type for comparison.

Results For a cytologic diagnosis with $22 \mathrm{Gpc}$, an adequate sample was obtained in $82.8 \%$ SST vs. $80.0 \%$ CST $(P=0.79)$. For a histologic diagnosis with $22 \mathrm{Gpc}$, an adequate sample was obtained in $70.4 \%$ SST vs. $69.0 \%$ CST ( $P$ $=0.91)$. A single pass with $22 \mathrm{Gpc}$ provided comparable results to a single pass with the $25 \mathrm{G}$ needle for a cytologic diagnosis; both were superior to a single $22 \mathrm{Gpc}$ pass for a histologic diagnosis. Two passes with the $25 \mathrm{G}$ needle provided a diagnostic specimen in $95.0 \%$ vs $81.4 \%$ with one pass using $22 \mathrm{Gpc}(P=0.01)$.

Conclusions No significant difference in diagnostic adequacy was observed between techniques for the $22 \mathrm{Gpc}$. Two passes with a $25 \mathrm{G}$ needle performed better than 1 pass with $22 \mathrm{Gpc}$. (NCT01598194)

Meeting presentations: Digestive Disease Week 2015

\section{Introduction}

EUS-FNA is the procedure of choice for diagnosing solid pancreatic masses and is most commonly performed with either a 22 or 25 gauge ( $G$ ) needle. The $25 \mathrm{G}$ needle has been suggested to be more sensitive for making a cytologic diagnosis of pancreatic malignancy $[1,2]$. The development of a $22 \mathrm{G}$ core needle [Procore ${ }^{\circledR}$ ] (pc) with reverse side bevel design may provide comparable or better material for making either a cytologic or histologic diagnosis with the potential for fewer passes, although studies thus far have produced mixed results [3]. The optimal technique for FNA with a $22 \mathrm{Gpc}$ needle is unclear but may affect specimen adequacy. Capillary suction technique (CST), which utilizes suction created by slow withdrawal of the stylet, has been suggested to enhance quality of the specimen and diagnostic adequacy versus standard suction technique (SST) [4-7].

The aims of this study were to evaluate the $22 \mathrm{Gpc}$ using SST and CST and compare diagnostic adequacy of $22 \mathrm{Gpc}$ with the standard $25 \mathrm{G}$ needle for solid pancreatic masses. 


\section{Patients and methods}

The study was approved by The University of Texas MD Anderson Cancer Center Institutional Review Board. (ClinicalTrials. gov identifier:NCT01598194) A single-center, prospective, randomized evaluation was performed. Sixty consecutive patients referred for diagnostic EUS-FNA of suspected solid pancreatic mass lesions on cross-sectional CT imaging were recruited from February 2012 to July 2013.

Each patient underwent 4 study passes. All patients underwent 2 passes with a standard $25 \mathrm{G}$ needle (Cook EchoTip ${ }^{\circledR}$ Ultra HD Endoscopic Ultrasound Needle, Cook Medical, Bloomington, IN, USA) for cytologic analysis; these passes were performed in uniform fashion using a stylet and full 10-cc suction via a transgastric (pancreas body/tail lesions) or transduodenal (pancreas head lesions) approach.

Patients were randomized into one of two groups for the $22 \mathrm{Gpc}$ passes as follows: The first group of 30 patients underwent 2 passes with a $22 \mathrm{Gpc}$ needle (Cook EchoTip ProCore ${ }^{\circledR}$ HD Ultrasound Biopsy Needle, Cook Medical, Bloomington, IN, USA) using SST; 1 pass for cytologic analysis followed by 1 pass for histologic analysis. The second group of 30 patients underwent 2 passes with the 22 Gpc needle using CST; 1 pass for cytologic analysis followed by 1 pass for histologic analysis. See Fig. 1 for definitions and description of study techniques.

The sequence of the 3 passes for cytological analysis (passes 1 to 3) was randomized by computer-generated assignment. Slides were prepared and labeled by individual pass in room by a trained cytotechnician. Slides were then transferred to a separate cytopathology lab for processing and preliminary intraprocedural interpretation. The last pass with the $22 \mathrm{Gpc}$ (pass 4) was always taken for histology and processed separately post-procedure. Any additional passes taken after preliminary cytological assessment were not included in the study analysis.

The main outcome measure of the study was the diagnostic adequacy of the 22 Gpc using SST and CST. The cytologic diagnostic adequacy of $22 \mathrm{Gpc}$ was also compared to the standard $25 \mathrm{G}$ needle. Diagnostic adequacy was defined as the ability to procure cytological aspirates or histological core tissue samples that were sufficient for diagnostic interpretation [3]. Two cytopathologists (SK, AS), blinded to needle type and technique, reviewed and graded all the study slides. Cytologic diagnostic adequacy of each pass was graded on a semiquantitative scale from 0 to 3 based on sample cellularity. A score of 2 (estimated cell count $>500$ cells) or 3 (estimated cell count $>1000$ cells) was considered adequate for diagnosis; a score of 3 was most desirable. A score $<2$ was considered inadequate. Histologic diagnostic adequacy was graded as either an adequate (score 2 or 3 ) or inadequate specimen for diagnosis.

Written informed consent was obtained from all study patients. Exclusion criterion included age $<18$ years, abnormal coagulation parameters, cystic lesions, extra-pancreatic lesions, and inaccessible or non-visualized lesions. All procedures were performed with monitored anesthesia care for sedation. Procedures were performed by four experienced endosonographers (BW, WR, MB, JL). A curved linear array echoendoscope (UCT-
Definition and description of study techniques

Standard Suction Technique (SST) 22 Gpc: Puncture of the lesion was performed with the inserted stylet, which was rapidly removed once lesion penetrated. Full suction was applied for approximately $15-30$ seconds using a $10-\mathrm{mL}$ syringe before at least 5-10 to-and-fro movements of the needle within the lesion as per manufacturer's instructions. Suction was released before the needle was withdrawn from the lesion to avoid aspiration of intestinal contaminant. The stylet + + - air was used to expel specimen. The stylet was used for all passes.

Capillary Suction Technique (CST) 22 Gpc: Puncture of the lesion was performed with the fully inserted stylet, which was slowly removed once lesion penetrated over approximately $15-30$ second with at least 5-10 to-and-fro movements of the needle within the lesion. No suction was applied otherwise. Stylet +/- air was used to expel specimen. The stylet was used for all passes.

Standard $25 \mathrm{G}$ technique: Puncture of the lesion was performed with the inserted stylet usually partially withdrawn at the tip. Upon successful penetration of the lesion, the stylet was then fully reinserted to clear the distal tip and then rapidly removed. Full suction was applied using a $10-\mathrm{mL}$ syringe before at least 5-10 to-and-fro movements of the needle within the lesion. Suction was released before the needle was withdrawn from the lesion to avoid aspiration of intestinal contaminant. The stylet $+/$ - air was used to expel specimen. The stylet was used for all passes.

Specimen preparation: Cytologic specimens were prepared on slides labeled by individual pass in room by a trained cytotechnician. Slides were then transferred to the cytopathology lab for processing. Air-dried smears were stained with Diff-Quik stain. Alcohol-stained smears were prepared by using Papanicolaou stain. Cell-block and histologic specimens were fixed in formalin, embedded in paraffin and then prepared in hematoxylin and eosin for subsequent evaluation after the procedure. Immunohistochemical or special staining was performed as appropriate.

Fig. 1 Definition and description of study techniques.

140P-AL5 Olympus Ltd, Tokyo, Japan) was used with an Aloka Pro-Sound SSD-Alpha 10 processor at $7.5 \mathrm{MHz}$ frequency. The lesion of interest was identified and assessed for location, size, solid echofeatures and regional vasculature. All patients, had follow-up within 2 weeks of the procedure. Information regarding adverse events (including but not limited to bleeding, pancreatitis, pain or infection) was obtained from electronic chart review. Data safety monitoring was also required as per IRB study protocol after the first 10 and 30 patients. There was no additional cost to the patient for the study needle.

\section{Statistical analysis}

Given the absence of available data for suction technique and this needle, novel at the time of study inception, a sample size calculation based on suction technique with the $22 \mathrm{Gpc}$ was not performed.

Sample size calculation for a comparison of the 2 different needle types was based on limited preliminary data for the 22 Gpc. McNemar's test was used for the sample size justification. The 2 standard FNA passes were assumed to provide diagnostic adequacy in at least $65 \%$ of patients [8]. The 2 pc 
- Table 1 Sample size calculation for comparison 22 Gpc vs $25 \mathrm{G}$ needle.

\begin{tabular}{|l|l|l|l|l|}
\hline $\begin{array}{l}\text { Estimated } \\
\text { Sample size }\end{array}$ & $\begin{array}{l}\text { Difference } \\
\text { in positive } \\
\text { proportions }\end{array}$ & $\begin{array}{l}\text { Proportion } \\
\text { Discordant }\end{array}$ & power & alpha \\
\hline 60 & 0.20 & 0.3 & 0.8 & 0.05 \\
\hline
\end{tabular}

needle passes were predicted to provide diagnostic adequacy in $85 \%$ of patients [ $9-12$ ]. Assuming a discordant proportion of $30 \%$, a sample size of 60 patients was calculated to achieve $80 \%$ power with an alpha at $5 \%$ ( $\triangleright$ Table 1 ).

Quantitative descriptive analyses were computed for all variables as appropriate. Frequencies or means were calculated for demographic and clinical characteristics and compared between SST and CST groups ( $\downarrow$ Table 2 ). We compared scores from individual passes with the $22 \mathrm{G}$ core needle by technique using Pearson's chi-squared test ( $\triangleright$ Table 3 ). Raw scores from all individual passes were tabulated ( $\triangleright$ Table 4$)$. Scores from in- dividual passes with the standard $25 \mathrm{G}$ needle were compared with the $22 \mathrm{G}$ core needle using McNemar's exact test ( $>$ Table 5). A $P$ value $<0.05$ was considered significant. All statistical analyses were performed using Stata software version 14 (StataCorp, College Station, TX).

\section{Results}

Seventy-three patients were enrolled. Thirteen were excluded after initial consent. Sixty consecutive patients participated. See $>$ Fig. 2 for a participant flow diagram.

A diagnosis was established in all cases. The final diagnosis was malignant in $86.7 \%$ in the SST group vs $96.7 \%$ in the CST group. Adenocarcinoma was the most common diagnosis overall $(75 \% ; 45 / 60)$ and in each group (73.3\% SST vs $76.7 \%$ CST). Patient and tumor characteristics of both groups are summarized in $>$ Table 2.

The scores from individual passes with the $22 \mathrm{Gpc}$ by technique are summarized in $\mathbf{T a b l e} 3$. With respect to our primary measure, no significant difference was observed in diagnostic

Table 2 Patient and tumor characteristics of Standard Suction (SST) vs. Capillary Suction (CST) group.

\begin{tabular}{|c|c|c|}
\hline & SST (group 1) $n=30$ & CST (group 2) $n=30$ \\
\hline Sex male, $n(\%)$ & $16(53.3)$ & $19(63.3)$ \\
\hline Mean age in years (range) & $65(31-78)$ & $61(24-85)$ \\
\hline Location mass pancreas head, n (\%) & $16(53.3)$ & $14(46.7)$ \\
\hline Mean size in millimeters (range) & $31(14-70)$ & $34(10-67)$ \\
\hline Hypoechoic, n (\%) & $27(90.0)$ & $26(86.7)$ \\
\hline \multicolumn{3}{|l|}{ Diagnosis } \\
\hline " Malignant, n (\%) & $26(86.7)$ & $29(96.7)$ \\
\hline - Adenocarinoma, n (\%) & $22(73.3)$ & $23(76.7)$ \\
\hline - Other, n (\%) & $8^{1}$ & $7^{2}$ \\
\hline
\end{tabular}

- Table 3 Comparison of diagnostic adequacy scores with standard suction technique (SST) versus capillary suction technique (CST) using a 22-gauge core needle.

\begin{tabular}{|c|c|c|c|c|c|c|}
\hline \multirow[b]{2}{*}{ All pancreas masses } & \multicolumn{3}{|l|}{ Cytology } & \multicolumn{3}{|l|}{ Histology } \\
\hline & SST & CST & $P$ value & SST & CST & $P$ value \\
\hline Score 0 or $1, n / N(\%)$ & $5 / 29(17.2)$ & $6 / 30(20.0)$ & & $8 / 27(29.6)$ & $9 / 29(31.0)$ & \\
\hline Score 2 or $3, \mathrm{n} / \mathrm{N}(\%)$ & $24 / 29(82.8)$ & $24 / 30(80.0)$ & 0.79 & $19 / 27(70.4)$ & $20 / 29(69.0)$ & 0.91 \\
\hline Score $3, \mathrm{n} / \mathrm{N}(\%)$ & $23 / 29(79.3)$ & $21 / 30(70.0)$ & 0.41 & & & \\
\hline \multicolumn{7}{|l|}{ Adenocarcinoma } \\
\hline Score 0 or $1, n / N(\%)$ & $4 / 22(18.2)$ & $5 / 23(21.7)$ & & $5 / 20(25.0)$ & $8 / 22(36.4)$ & \\
\hline Score 2 or $3, \mathrm{n} / \mathrm{N}(\%)$ & $18 / 22(81.8)$ & $18 / 23(78.3)$ & 0.77 & $15 / 20(75.0)$ & $14 / 22(63.6)$ & 0.43 \\
\hline Score $3, \mathrm{n} / \mathrm{N}(\%)$ & $17 / 22(77.3)$ & $17 / 23(73.9)$ & 0.79 & & & \\
\hline $\begin{array}{l}\text { Score } 0 \text { or } 1=\text { non-diagno } \\
\text { Score } 2 \text { or } 3=\text { diagnostic } \\
P \text { values calculated using }\end{array}$ & uared test. & & & & & \\
\hline
\end{tabular}


- Table4 Diagnostic adequacy scores from individual FNA passes.

\begin{tabular}{|c|c|c|c|c|}
\hline All pancreas masses & Pass $1(25 G) n=60$ & Pass $2(25 G) n=60$ & Pass $3(22 \mathrm{Gpc}) n=59^{1}$ & Pass $4(22 \mathrm{Gpc}) n=56^{2}$ \\
\hline Score 2-3 & $49(81.7 \%)$ & $52(86.7 \%)$ & $48(81.4 \%)$ & $39(69.6 \%)$ \\
\hline Score $0-1$ & $11(18.3 \%)$ & $8(13.3 \%)$ & $11(18.6 \%)$ & $17(30.4 \%)$ \\
\hline Score 3 & $38(63.3 \%)$ & $37(61.7 \%)$ & $44(74.6 \%)$ & \\
\hline Score $0-2$ & $22(36.7 \%)$ & $23(38.3 \%)$ & $15(25.4 \%)$ & \\
\hline Adenocarinoma & Pass $1(25 G) n=45$ & Pass $2(25 G) n=45$ & Pass $3(22 G p c) n=45$ & Pass $4(22 G p c) n=42^{3}$ \\
\hline Score $2-3$ & $37(82.2 \%)$ & $39(86.7 \%)$ & $36(80.0 \%)$ & $29(69.0 \%)$ \\
\hline Score $0-1$ & $8(17.8 \%)$ & $6(13.3 \%)$ & $9(20.0 \%)$ & $13(31.0 \%)$ \\
\hline Score 3 & $29(64.4 \%)$ & $27(60.0 \%)$ & $34(75.6 \%)$ & \\
\hline Score $0-2$ & $16(35.6 \%)$ & $18(40.0 \%)$ & $11(24.4 \%)$ & \\
\hline $\begin{array}{l}\text { Pass } 1-3 \text { for cytology (se } \\
\text { Score } 0 \text { or } 1=\text { non-diagno } \\
\text { Score } 2 \text { or } 3=\text { diagnostic } \\
1 \text { not done in } 1 \text { pt } \\
2 \text { not done in } 4 \text { pts } \\
{ }^{3} \text { not done in } 3 \text { pts }\end{array}$ & ized); Pass 4 for histolog & & & \\
\hline
\end{tabular}

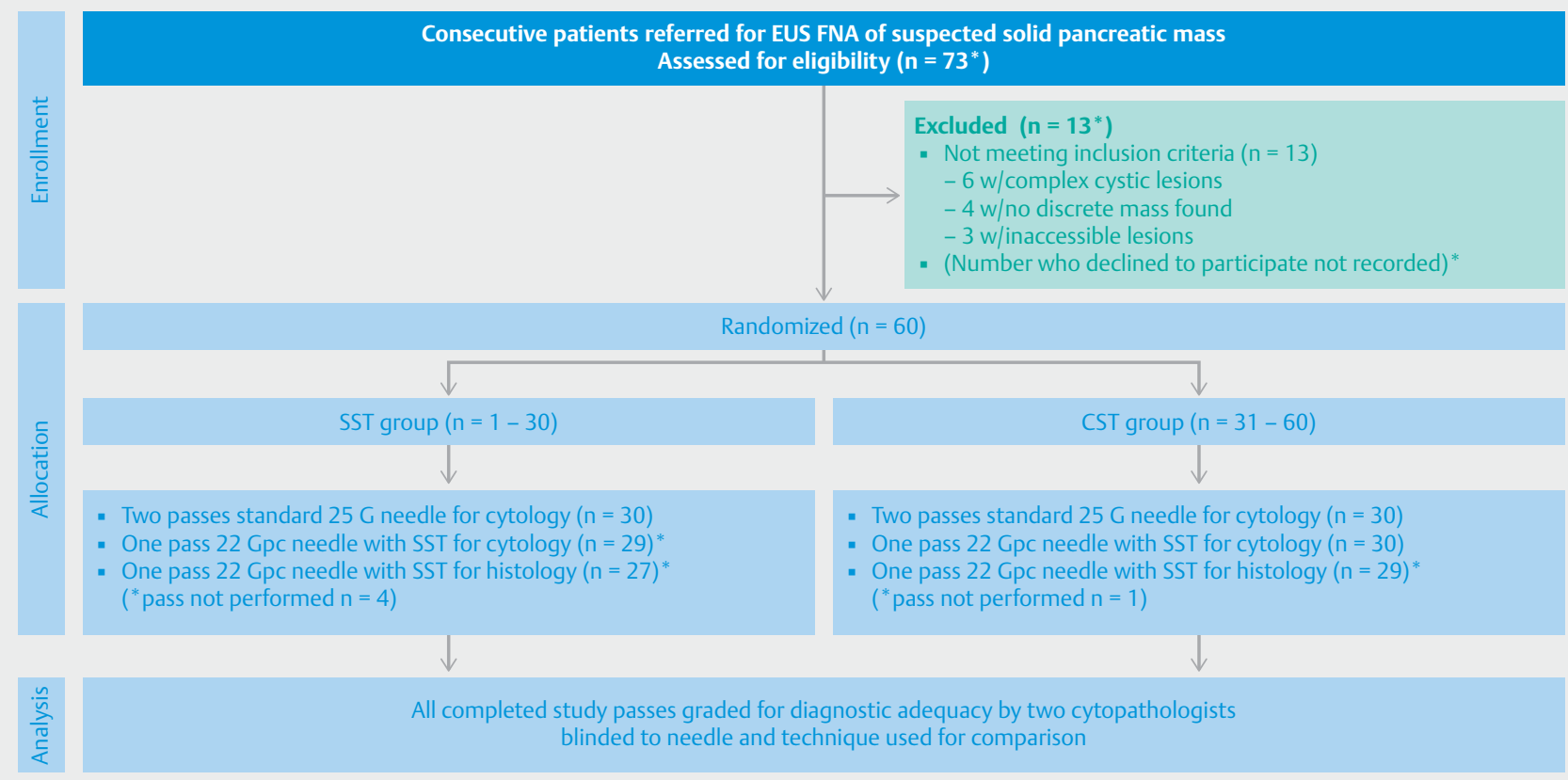

Fig. 2 Participant flow diagram.

adequacy between SST and CST techniques for making a cytologic or histologic diagnosis of solid pancreatic masses using the $22 \mathrm{Gpc}$.

For a cytologic diagnosis with the $22 \mathrm{Gpc}$, a score of 2 to 3 was obtained in $82.8 \%$ with SST vs $80 \%$ with CST $(P=0.79)$; a score of 3 was obtained in $79.3 \%$ with SST vs $70 \%$ with CST $(P=0.41)$. FNA was not done in 1 patient with the $22 \mathrm{Gpc}$ with SST because of operator difficulty advancing the needle into a pancreatic head lesion from the transduodenal position.
For a histologic diagnosis with the $22 \mathrm{Gpc}$, an adequate sample for diagnosis was obtained in $70.4 \%$ with SST vs $69.0 \%$ with CST $(P=0.91)$. FNB was not done in 3 patients with the $22 \mathrm{G} \mathrm{pc}$ with SST because of operator difficulty advancing the needle into a pancreatic head lesion from the transduodenal position, bending of needle after first pass, and need for additional passes after preliminary cytologic analysis. FNB was unsuccessful in 1 patient with the $22 \mathrm{G}$ pc with CST because no specimen was obtained. 
- Table 5 Comparison of a single pass with $22 \mathrm{G}$ core needle and the standard $25 \mathrm{G}$ needle for cytologic diagnosis.

\begin{tabular}{|c|c|c|c|c|c|c|c|}
\hline All pancreas masses & $22 \mathrm{Gpc}^{1}$ & $25 \mathrm{G}$ pass one & $P$ & $25 \mathrm{G}$ pass two & $P$ & $25 \mathrm{G}$ best of 2 passes & $P$ \\
\hline Score 2 or $3 \mathrm{n} / \mathrm{N}(\%)$ & $48 / 59(81.4)$ & $49 / 60(81.7)$ & 1.00 & $52 / 60(86.7)$ & 0.51 & $57 / 60(95.0)$ & $0.02^{2}$ \\
\hline Score 3 & $44 / 59(74.6)$ & $38 / 60(63.3)$ & 0.09 & $37 / 60(61.7)$ & 0.04 & $45 / 60(75.0)$ & 0.96 \\
\hline Adenocarcinoma & $22 \mathrm{Gpc}^{1}$ & $25 \mathrm{G}$ pass one & $P$ & $25 \mathrm{G}$ pass two & $P$ & $25 \mathrm{G}$ best of 2 passes & $P$ \\
\hline Score 2 or 3 & $36 / 45(80.0)$ & $37 / 45(82.2)$ & 1.00 & $39 / 45(86.7)$ & 0.45 & $43 / 45(95.6)$ & $0.04^{3}$ \\
\hline Score 3 & $34 / 45(75.6)$ & $29 / 45(64.4)$ & 0.18 & $27 / 45(60.0)$ & 0.02 & $34 / 45(75.6)$ & 1.00 \\
\hline \multicolumn{8}{|c|}{$\begin{array}{l}P \text { values calculated using McNemar's exact test } \\
1 \text { reference group for comparison with different passes with } 25 \mathrm{G} \\
2 \text { difference } 13.6(95 \% \mathrm{CI} 1.9,25.2) \\
{ }^{2} \text { difference } 15.6(95 \% \mathrm{Cl} 1.1,30.0)\end{array}$} \\
\hline
\end{tabular}

For pancreas adenocarcinoma alone, no significant difference was observed between techniques for specimen adequacy to make a cytologic or histologic diagnosis using the $22 \mathrm{Gpc}$. For a cytologic diagnosis of adenocarcinoma with the $22 \mathrm{Gpc}$, a score of 2 to 3 was obtained in $81.8 \%$ with SST vs $78.3 \%$ with CST $(P=0.77)$; a score of 3 was obtained in $77.3 \%$ with SST vs $73.9 \%$ with CST $(P=0.79)$. For a histologic diagnosis of adenocarcinoma with the $22 \mathrm{Gpc}$, an adequate sample was obtained in $75.0 \%$ with SST vs $63.6 \%$ with CST $(P=0.43)$. - Table 3 .

The cumulative raw scores from all individual FNA passes are provided in $>$ Table 4. An overall comparison of scores with the $22 \mathrm{Gpc}$ vs the standard $25 \mathrm{G}$ for a cytological diagnosis of pancreas lesions is summarized in $>$ Table 5 . No significant difference was observed for a score of 2 to 3 between the $22 \mathrm{Gpc}$ and either of the 2 individual passes with the $25 \mathrm{G}$ needle for all solid pancreatic masses [ $81.4 \%$ vs $81.7 \%(P=1)$ and $86.7 \%$ $(P=0.51)]$ or adenocarcinoma $[80.0 \%$ vs $82.2 \%(P=1.00)$ and $86.7 \%(P=0.45)]$. A single pass with the $22 \mathrm{Gpc}$ did yield a higher percent of score 3 than both individual $25 \mathrm{G}$ needle passes for all pancreatic masses [74.6\% vs 63.3\% $(P=0.09)$ and $61.7 \%(P=$ $0.04)$ ] and adenocarcinoma [75.6\% vs $64.4 \%(P=0.18)$ and $60 \%$ $(P=0.02)]$. No significant difference was observed between the 2 standard $25 G$ passes $(P>0.05)$. However, 2 passes with the $25 \mathrm{G}$ needle performed significantly better than a single pass with the $22 \mathrm{Gpc}$ for a cytologic diagnosis with a score of 2 to 3 for all pancreatic masses [95\% vs $81.4 \%(P=0.02)$ ] and adenocarcinoma [95.6\% vs $80.0 \%(P=0.04)$ ]. Furthermore, a highquality cytologic specimen with a score of 3 was obtained just as often with 2 passes with a $25 \mathrm{G}$ needle as with 1 pass with a $22 \mathrm{Gpc}$ for all pancreatic masses $[75.0 \%$ vs $74.6 \%(P=0.96)]$ and adenocarcinoma $[75.6 \%$ vs $75.6 \%(P=1)]$. A single FNA pass with either the $25 \mathrm{G}$ or $22 \mathrm{Gpc}$ needle for a cytological diagnosis was superior to a single FNB pass with $22 \mathrm{Gpc}$ pass for a histologic diagnosis ( $>$ Table $\mathbf{3}$ ).

No adverse events were observed.

\section{Discussion}

Our results show no apparent difference in diagnostic adequacy between SST and CST with the 22 Gpc needle for either a cytologic or histologic diagnosis of solid pancreatic lesions or ade- nocarcinoma. A single pass with the $22 \mathrm{Gpc}$ provided an adequate specimen for cytologic diagnosis approximately $81 \%$ and histologic diagnosis $70 \%$ of the time irrespective of suction technique. Overall, a single pass with the $22 \mathrm{Gpc}$ was inferior to $225 \mathrm{G}$ passes, which procured a sample sufficient for cytologic diagnosis in $81.4 \%$ vs $95.0 \%$ respectively.

Although EUS-FNA is the current standard of care for sampling pancreatic mass lesions, the technique is not without its limitations. The rates of diagnostic adequacy have been reported to be variable $[3,13-17]$. Multiple studies have been performed looking at the optimal technique, needle size, and number of passes with mixed results reported across the literature depending largely on the nature and location of the lesion.

EUS-FNA of solid pancreatic masses is most commonly performed with either a 22 or $25 \mathrm{G}$ needle. At present the standard $25 \mathrm{G}$ needle is the preferred and most sensitive needle in most circumstances for obtaining adequate material for a cytologic diagnosis of solid pancreas lesions especially for adenocarcinoma, and especially when rapid on-site evaluation (ROSE) is available [1,2]. The smaller, more flexible $25 \mathrm{G}$ needle offers a number of advantages including technical ease of use, and provision of a more cellular aspirate with less blood contamination. Still, some lesions will require extra material or larger specimens with intact architecture for diagnosis or ancillary testing. The limited availability of ROSE and other factors including the demand for additional material for molecular studies in the era of personalized cancer therapy has led the impetus for the development of better needles that allow for fewer passes.

The Procore ${ }^{\circledR}(\mathrm{pc})$ needle with reverse side bevel design has been reported to procure comparable material for either a histologic or cytologic diagnosis with the potential for fewer passes [3]. Initial feasibility studies with the larger 19Gpc needle were promising for obtaining histologic specimens showing an increase in diagnostic adequacy and accuracy of up to $15 \%$ compared to FNA with the standard needle [10]. However, inconsistent results, technical sampling limitations and an inferior cytologic yield led to a $22 \mathrm{Gpc}$ and $25 \mathrm{Gpc}$ version. Subsequent evaluation of these core needles has also been discordant $[9,11,18-25]$. A recent meta-analysis did not demonstrate a significant difference between pc and standard FNA needles for sample adequacy, diagnostic accuracy or acquisi- 
tion of a core specimen, although the pc needle may establish the diagnosis with fewer passes [3]. The absence of an established standardized technique may explain the inconsistency.

The optimal technique for FNA with core needles is unknown but may affect adequacy. Studies looking at the application of suction with standard FNA needles have demonstrated mixed results [26-31]. In general, suction increases cellularity but also contamination with blood, which may limit cytological interpretation. Although technical guidelines recommend the application of suction for EUS-FNA of solid masses [32, 33], the use of suction and how it is performed during FNA varies widely. Normal suction is generally performed using a $10-\mathrm{mL}$ syringe and may be applied continuously or intermittently. Many endosonographers will adjust suction strength depending on the nature of the lesion and aspirate. Low or no suction is preferred if the aspirate is bloody. Suction is preferred if the initial aspirate is scant, and may increase cellularity in fibrotic lesions [26].

CST, which utilizes capillary aspiration created by slow withdrawal of the stylet, has been suggested in limited retrospective studies to enhance quality of the specimen obtained for diagnosis versus SST [4-7]. The main rationale for improved sampling with CST versus SST is the procurement of a less bloody and reportedly larger aspirate with reduced tissue trauma.

Chen et al. was first to report that CST may increase diagnostic yield over SST for FNA of solid pancreatic lesions using a standard needle [4]. In their retrospective analysis of 91 patients who underwent sampling with either CST or SST (mean 3.4 passes), the sensitivity was higher for the CST group ( $94 \%$ vs $80 \%$ ) although statistical significance was not reached. Quality of the cytology specimen was better with CST (55\% vs $33 \%$ ). Needle size was not specified.

Nakai et al., in their retrospective analysis of 97 patients who underwent either CST or SST using standard $25 \mathrm{G}$ and $22 \mathrm{G} \mathrm{FNA}$ needles, found that CST was associated with less blood contamination, lower scores for cellularity but potentially increased diagnostic yield (sensitivity $90.0 \%$ vs $67.9 \%$ ), especially with the $25 G$ needle [5].

The potential benefits of the core needle venting mechanism and/or cutting action associated with the reverse side bevel design on suction is unclear. Kothari et al evaluated different techniques with a $22 \mathrm{Gpc}$ needle in a porcine pancreas model, and found that CST provided superior tissue adequacy scores compared to full (10 cc) or half suction [6]. Iwashita et al. reported their retrospective results with a $25 \mathrm{Gpc}$ needle in 60 consecutive patients who underwent a median number of 4 passes with either SST or CST using the $25 \mathrm{Gpc}$. The first 20 were evaluated with SST, the last 40 with CST. A histological diagnosis was established in $90 \%$ CST vs $55 \%$ SST $(P<0.01)$. A cytological diagnosis was established in $92 \%$ CST vs $70 \%$ SST $(P<0.05)$. Overall diagnostic yields were $75 \%$ and $95 \%$ in SST and CST, respectively $(P<0.05)[7]$.

Our study with the $22 \mathrm{Gpc}$ did not demonstrate any significant differences in CST versus SST for either the cytologic or histologic diagnostic adequacy of solid pancreatic masses. One possible explanation for this finding may be related to our study design. Unlike previous retrospective studies, our prospective evaluation allowed for a well-controlled standardized comparison of both techniques $[9,10,23]$. Our study also enabled direct comparison of the $22 \mathrm{Gpc}$ with the standard $25 \mathrm{G}$ needle in the same lesion in a crossover controlled fashion. Similar to previous studies, a high-quality single-pass diagnostic cytologic specimen was provided with the $22 \mathrm{Gpc}$ needle and was comparable to a single pass with the $25 \mathrm{G}$ needle for solid pancreatic lesions. However 2 passes with a $25 \mathrm{G}$ needle (approaching $95 \%$ ) were superior to a single pass with the $22 \mathrm{Gpc}$ [24].

Vanbiervliet et al found no difference in diagnostic accuracy between $122 \mathrm{Gpc}$ pass and 2 standard 22 G needle passes for solid pancreatic masses in their prospective study of 80 patients using SST only ( $90 \%$ vs $92.5 \% ; P=0.68$ ) [23]. Unlike our study, they did not differentiate individual passes with the standard FNA needle and samples were separately processed for liquid based cytology and cell-block preparation (no smears). Interestingly the overall sample quality proved better for the standard needle than $22 \mathrm{Gpc}$ in comparison to our study which showed the $22 \mathrm{Gpc}$ to have a higher percentage of optimal scores (score 3).

Only 1 other study to date has compared the $22 \mathrm{Gpc}$ needle to the standard $25 \mathrm{G}$ needle. Berdoza et al also found no significant difference in diagnostic adequacy between these two needles in their retrospective analysis of 56 patients (81.9\% vs 73.8\%; $(P=0.37)$ [34]. Similar to our study, both needles were tested in the same lesion, however a lower mean number of passes (1.7 vs 3.5) was noted with the core needle. Acknowledged limitations included absence of standardized technique, non-randomized sequence and non-blinded pathologist.

In our study, sampling adequacy provided by a single pass with the 22 Gpc for a histology based diagnosis at $~ 70 \%$ was inferior to cytology based results with either needle, consistent with the findings of others at $\sim 63-66 \%[23,24]$. Combining the cytologic and histologic results from a single pass with $22 \mathrm{Gpc}$ would not appear to improve diagnostic adequacy over two passes with a $25 \mathrm{G}$ needle. Similar findings were observed in a recent comparison of the $22 \mathrm{Gpc}$ vs $25 \mathrm{Gpc}$ [25]. Although we expect incremental yield with a second $22 \mathrm{Gpc}$ pass comparable to at least $225 \mathrm{G}$ passes, our findings would argue against existing data that a lower number of passes to establish a diagnosis is achieved with the 22 Gpc.

The main strengths of our study include its prospective and controlled design. FNA techniques were standardized. The scoring scale used to evaluate our primary outcomes, provided a uniform platform to grade and compare specimens by the cytopathologists, who were blinded to needle type and suction technique. The sequence of passes for cytology was also randomized given that it may be possible for an initial needle pass to impact the quality of the sample obtained by a subsequent pass. The direct comparison of needles within the same lesion in a randomized crossover fashion was an important and unique strength of our study and enabled us to reduce bias caused by variation in the nature of any given lesion, which may affect sampling. Ideally an extra 22 Gpc study pass for both cytology and histology with each technique would have enabled for a more uniform comparison, however it was important to minimize the risk of unnecessary passes. Although many studies 
have demonstrated the safety of multiple passes, we limited our evaluation to 4 .

Our study has several limitations. The study was conducted at a single high-volume tertiary cancer center and our results may not be generalizable to other practices or those without ROSE [35]. The scale used to evaluate our primary outcomes has not been validated, although no universal validated cytological scoring system currently exists. A formal sample size calculation was not performed for suction technique based on limited data with the $22 \mathrm{Gpc}$ needle at the time of study inception, as well as absence of a definitive difference in suction technique with standard needles. Nonetheless, we felt our sample size was adequate to see if an important difference might exist. Although the possibility of a type 2 error exists, no trend towards a difference in technique was apparent in our study. Calculated sample size for comparisons made between needle type appear underpowered based on the high diagnostic adequacy and concordance of our findings and what proved to be a conservative sensitivity rate used for two passes with the standard $25 \mathrm{G}$ needle. A sample size of approximately 234 would have been needed if we reduced the estimated detectable difference from $20 \%$ to $10 \%$ respectively. Unfortunately, resources for study needles were limited. Arguably a larger study to prove a smaller difference may be of limited practical or clinical utility. Although our study included all solid pancreatic masses, $75 \%$ were adenocarcinoma and our results will not be applicable for all lesions. Our study did not allow for quantification of specimen size. Although measurement of volume or weight with cell block preparation or liquid based cytology could have facilitated this, an immediate diagnosis with slide preparation was essential to initiate treatment and avoid non-diagnostic procedures. A low but nonetheless higher incidence of technical failure was observed with the $22 \mathrm{Gpc}$ versus the $25 \mathrm{G}$ needle, all of which occurred with transduodenal sampling of pancreatic head lesions.

\section{Conclusions}

In conclusion, our results showed no difference in diagnostic adequacy between suction technique for the $22 \mathrm{Gpc}$ needle for either a cytologic or histologic diagnosis of solid pancreatic masses or adenocarcinoma. A single $22 \mathrm{Gpc}$ pass was inferior to the high diagnostic adequacy of 2 standard $25 \mathrm{G}$ passes for a cytologic diagnosis and inadequate for a consistent histologic diagnosis contrary to existing studies advocating the potential for fewer potential passes with the core needle. Although a single 22 Gpc pass may potentially provide a more optimal specimen, it seems likely that at least 2 passes are needed to optimize diagnostic adequacy, especially without ROSE. Larger studies may verify our results, although experience thus far indicates that no one needle or technique fits all pancreatic solid lesions.

\section{Competing interests}

\section{None}

\section{References}

[1] Madhoun MF, Wani SB, Rastogi A et al. The diagnostic accuracy of $22 \mathrm{G}$ and $25 \mathrm{G}$ needles in endoscopic ultrasound-guided fine needle aspiration of solid pancreatic lesions: a meta analysis. Endoscopy 2013; 45: 86-92

[2] Affolter KE, Schmidt RL, Matynia AP et al. Needle size has only a limited effect on outcomes in EUS-guided fine needle aspiration: a systematic review and meta-analysis. Dig Dis Sci 2013; 58: 1026-1034

[3] Bang JY, Hawes R, Varadarajulu S. A meta-analysis comparing ProCore and standard fine-needle aspiration needles for endoscopic ultrasound-guided tissue acquisition. Endoscopy 2016; 48: 339-349

[4] Chen AM, Park WG, Friedland S etal. Endoscopic ultrasound-guided fine needle aspiration versus fine needle capillary sampling biopsy of pancreatic solid lesions: does technique matter? Gastrointest Endosc 2011; 73: (Suppl. 04): AB331(1396)

[5] Nakai $\mathrm{Y}$, Isayama $\mathrm{H}$, Chang $\mathrm{KJ}$ et al. Slow pull versus suction in endoscopic ultrasound-guided fine-needle aspiration of pancreatic solid masses. Dig Dis Sci 2014; 59: 1578-1585

[6] Kothari S, Chen AM, Pai R et al. Comparison of EUS-guided pancreas biopsy techniques using the Procore needle. Gastrointes Endosc 2012; 75: AB145

[7] Iwashita T, Nakai Y, Samarasena JB et al. Endoscopic ultrasoundguided fine needle aspiration and biopsy (EUS-FNAB) using a novel 25-gauge core biopsy needle: optimizing the yield of both cytology and histology. Gastrointest Endosc 2012; 75: AB183

[8] Savides TJ, Donohue M, Hunt G et al. EUS-guided FNA diagnostic yield of malignancy in solid pancreatic masses: a benchmark for quality performance measurement. Gastrointest Endosc 2007; 66: 277-282

[9] Bang JY, Hebert-Magee S, Trevino J et al. Randomized trial comparing the 22-gauge aspiration and 22-gauge biopsy needles for EUS-guided sampling of solid pancreatic mass lesions. Gastrointest Endosc 2012; 76: $321-327$

[10] Iglesias-Garcia J, Poley JW, Larghi A et al. Feasibility and yield of a new EUS histology needle: results from a multicenter, pooled, cohort study. Gastrointest Endosc 2011; 73: 1189-1196

[11] Giovannini MM, Genevieve MM, Iglesias-Garcia J et al. Prospective multicenter evaluation of a novel 22-G echo-tip Procore histology EUS-needle in patients with a solid pancreatic mass. Gastrointest Endosc 2011; 73: AB152 - AB153 (abstract)

[12] Levy MJ, Wiersema MJ. EUS-guided Trucut biopsy. Gastrointest Endosc 2003; 57: 417-426

[13] Hartwig W, Schneider L, Diener MK et al. Preoperative tissue diagnosis for tumors of the pancreas. Br J Surg 2009; 96: 5-20

[14] Hewitt MJ, McPhail MJ, Possamai L et al. EUS-guided FNA for diagnosis of solid pancreatic neoplasms: a meta-analysis. Gastrointest Endosc 2012; 75: 319-331

[15] Puli SR, Bechtold MK, Buxbaum JL et al. How good is endoscopic ultrasound-guided fine-needle aspiration in diagnosing the correct etiology for a solid pancreatic mass? A meta-analysis and systematic review Pancreas 2013; 42: 20 - 26

[16] Chen G, Liu S, Zhao Y et al. Diagnostic accuracy of endoscopic ultrasound guided fine-beedle aspiration for pancreatic cancer: a meta-analysis. Pancreatology 2013; 13: 298-304

[17] Wiersema M], Vilmann P, Giovannini M et al. Endosonography-guided fine-needle aspiration biopsy: diagnostic accuracy and complication assessment. Gastroenterology 1997; 112: 1087-1095

[18] Iglesias-Garcia JA, Larino-Noia J, Dominguez-Munoz E. Sa1563 Differential diagnosis of solid pancreatic masses: Do procore histology needles improve the diagnostic yield of standard cytology needles? Gastrointest Endosc 2012; 75: AB2f03 
[19] Nguyen N. Sa1518 EUS guided fine needle core biopsy versus aspiration for upper gastrointestinal mass lesions: A randomized trial. Gastrointest Endosc 2012; 75: AB188

[20] Huci T, Wee E, Anuradha S et al. Feasibilty and efficiency of a new $22 \mathrm{G}$ core needle: a prospective comparison study. Endoscopy 2013; 45: $792-798$

[21] Larghi A, Iglesias-Garcia J, Poley JW et al. Feasibility and yield of a novel 22-gauge histology EUS needle in patients with pancreatic masses: a multicenter prospective cohort study. Surg Endosc 2013; 45: $3733-3738$

[22] Witt BL, Adler DG, Hilden K et al. A comparative needle study: EUSFNA procedures using the HD ProCore ${ }^{\mathrm{TM}}$ and EchoTip ${ }^{\circledR} 22$-gauge needle types. Diagn Cytopathol 2013; 41: 1069-1074

[23] Vanbiervliet G, Napoleon B, Saint Paul MC et al. Core needle versus standard needle for endoscopic ultrasound guided biopsy of solid pancreatic masses: a randomized crossover study. Endoscopy 2014; 46: $1063-1070$

[24] Iwashita T, Nakai Y, Samarasena JB et al. High single-pass diagnostic yield of a new 25-gauge core biopsy needle for EUS-guided FNA biopsy in solid pancreatic lesions. Gastrointest Endosc 2013; 77: 909-915

[25] Lee YN, Moon JH, Kim HK et al. Core biopsy needle versus standard aspiration needle for endoscopic ultrasound-guided sampling of solid pancreatic masses: a randomized parallel group-study. Endoscopy 2014; 46: $1056-1062$

[26] Puri R, Vilmann P, Saftoiu A et al. Randomized controlled trial of endoscopic ultrasound-guided fine needle sampling with or without suction for better cytological diagnosis. Scand J Gastroenterol 2009; 44: $499-504$

[27] Larghi A, Noffsinger A, Dye CE et al. EUS-guided fine needle tissue acquisition by using high negative pressure suction for the evaluation of solid masses: a pilot study. Gastrointest Endosc 2005; 62: 768 - 774
[28] Lee JK, Choi LH, Lee KH et al. A prospective, comparative trial to optimize sampling techniques in EUS-guided FNA of solid pancreatic masses. Gastrointest Endosc 2013; 77: 745 - 751

[29] Kudo T, Kawakami H, Hayashi T et al. High and low negative pressure suction techniques in EUS-guided fine-needle tissue acquisition by using 25-gauge needles: a multicenter, prospective, randomized, controlled trial. Gastrointest Endosc 2014; 80: 1030 - 1037

[30] Kundu S, Conway J, Gilbert K et al. Suctiuon or no suction? Interval results from an ongoing prospective, partially blind randomized trial of endoscopic ultrasound (EUS) guided fine needle aspiration of solid lesions Gastrointest Endosc 2009; 69: S248

[31] Wani S. Basic techniques in endoscopic ultrasound-guided fine needle aspiration: Role of a sytlet and suction. Endosc Ultrasound 2014; 3: $17-21$

[32] Wani S, Muthusamy R, Komanduri S. EUS-guided tissue acquisition: an evidence-based approach (with videos). Gastrointest Endosc 2014; 80: 939-959

[33] Polkowski M, Larghi A, Weynand B et al. Learning, techniques, and complications of endoscopic ultrasound (EUS)-guided sampling in gastroenterology: European Society of Gastrointestinal Endoscopy (ESGE) Technical Guideline. Endoscopy 2012; 44: 190 - 206

[34] Berzosa M, Villa N, El Serag HB et al. Comparison of endoscopic ultrasound guided 22-gauge core needle with standard 25-gauge fineneedle aspiration for diagnosis solid pancreatic lesions. Endosc Ultrasound 2015; 4: $28-33$

[35] Iglesias-Garcia J, Dominguez-Munoz JE, Abdulkader I et al. Influence of on-site cytopathology evaluation on the diagnostic accuracy of endoscopic ultrasound-guided fine needle aspiration (EUS-FNA) of solid pancreatic masses. Am J Gastroenterol 2011; 106: 1705-1710 